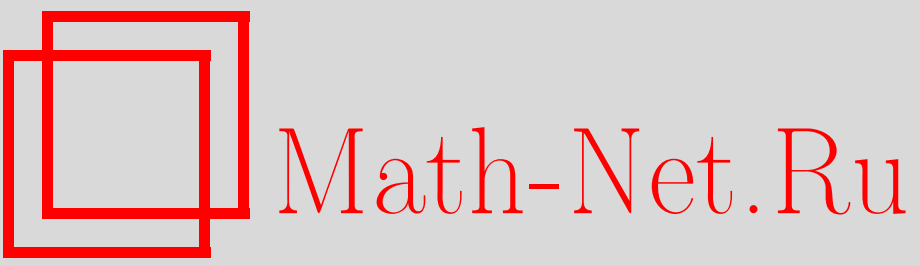

И. Н. Володин, М. В. Понфиленок, Уточняющие аппроксимации бетараспределения при малых значениях параметров, Теория вероятн. и ее примен., 2009, том 54, выпуск 3, 573-579

DOI: https://doi.org/10.4213/tvp2811

Использование Общероссийского математического портала Math-Net.Ru подразумевает, что вы прочитали и согласны с пользовательским соглашением

http: //www . mathnet.ru/rus/agreement

Параметры загрузки :

IP : 52.90 .164 .192

26 апреля 2023 г., 13:40:33 
18. Алексеев В.Г. О некоторых свойствах статистических оценок старших спектральных плотностей. - Теория вероятн. и ее примен., 1990, т. 35, в. 3, с. 431-437.

19. Алексеев В. Г. Биспектральный анализ стационарных случайных процессов: выборки большого объема. - Автометрия, 1990, № 5, с. 23-28.

20. Алексеев В. Г. О некоторых важнейших свойствах периодограмм старших порядков. - Теория вероятн. и ее примен., 1995, т. 40, в. 3, с. 481-494.

21. Алексеев В. Г. Новые модификации периодограмм второго и третьего порядков. Теория вероятн. и ее примен., 1997, т. 42, в. 4, с. 657-667.

22. Алексеев В.Г. О непараметрических методах прикладного биспектрального анализа. - Автометрия, 2006, т. 42, № 1, с. 13-22.

23. Ширяев А.Н. Вероятность-1. М.: МЦНМО, 2004, 519 с.

24. Алексеев В. Г. Об оценках триспектральной плотности стационарного случайного процесса. - Проблемы передачи информации, 1990, т. 26, № 1, с. 12-18.

25. Алексеев В. Г. Триспектральный анализ стационарных случайных процессов. Выборки большого объема. - Проблемы передачи информации, 1991, т. 27, №2, c. $69-74$.

26. Алексеев В.Г. Новая модификация кумулянтной периодограммы четвертого порядка. - Проблемы передачи информации, 1999, т. 35, № 3, с. 48-53.

27. Драган Я. П., Рожков В. А., Яворский И. Н. Методы вероятностного анализа ритмики океанологических процессов. Л.: Гидрометеоиздат, 1987, 319 с.

28. Рытов С. М. Введение в статистическую радиофизику, ч. I. М.: Наука, 1976, $494 \mathrm{c.}$

29. Яглом А. М. Периодически коррелированный случайный процесс. - Теория вероятностей и математическая статистика. Энциклопедия. Гл. ред. Ю. В. Прохоров. М.: Большая Российская энциклопедия, 1999, с. 451.

30. Алексеев В.Г. О свойствах симметрии старших спектральных плотностей стационарных и периодически нестационарных случайных процессов. - Проблемы передачи информации, 1987, т. 23, № 3, с. 48-53.

31. Алексеев В.Г. О свойствах симметрии старших спектральных плотностей периодически нестационарных случайных процессов. - Доклады по математике и ее приложениям, 1988, т. 2, № 4, с. 480-483.

32. Алексеев В. Г. Эмпирический спектральный анализ периодически коррелированных случайных процессов. Альтернативный подход. - Проблемы передачи информации, 1997, т. 33, № 4, с. 61-69.

33. Алексеев В.Г. Некоторые вопросы статистического анализа периодически коррелированных временных рядов. - Изв. РАН. Сер. Физика атмосферы и океана, 2005, т. 41, № 1, с. 125-130.

Поступила в редакцию

10.VIII. 2005

Исправленный вариант

12.II.2007

(c) 2009 г. $\quad$ ВОЛОДИН И. Н. ${ }^{*}$, ПОНФИЛЕНОК М. Н.*

\section{УТОЧНЯЮЩИЕ АППРОКСИМАЦИИ БЕТА-РАСПРЕДЕЛЕНИЯ ПРИ МАЛЫХ ЗНАЧЕНИЯХ ПАРАМЕТРОВ}

\footnotetext{
Разрабатывается метод последовательного уточнения аппроксимации бета-распределения при малых значениях параметров, предложенной в работе [4]. Находится асимптотика ошибки аппроксимации в терминах равномерного расстояния между распределениями.
}

* Казанский государственный университет, факультет вычислительной математики и кибернетики, кафедра математической статистики, 420008 Казань, Россия; e-mail: igor.volodin@ksu.ru 
Ключевые слова и фразы: бета-распределение, малые значения параметров, аппроксимация распределения, ошибка аппроксимации.

1. Введение. Асимптотическое исследование бета-распределения

$$
I(x)=I(x ; \alpha, \beta)=\frac{1}{\mathrm{~B}(\alpha, \beta)} \int_{0}^{x} t^{\alpha-1}(1-t)^{\beta-1} d t,
$$

$0 \leqslant x \leqslant 1, \alpha>0, \beta>0$, при $\varepsilon=\alpha+\beta \rightarrow 0$ было предпринято в работе [4] в связи с изданием на русском языке [3] таблиц К. Пирсона [2]. Интерполяция табличных данных при малых значениях параметров вызывала большие трудности и делала невозможным использование таблиц во всей области параметрического пространства. Естественно, в настоящее время вместо таблиц целесообразнее использовать компьютер, обращаясь, скажем, к пакету Mathematica. Тем не менее, применение таких асимптотик может оказаться полезным при решении ряда задач байесовского подхода, когда статистик имеет дело с последовательностями статистических экспериментов в рамках схемы Бернулли, причем вероятность успеха может принимать только значения, близкие к нулю или к единице (об использовании бета-распределения в рамках байесовского подхода см. [1, гл. 13]).

Для решения таких задач в работе [4] была построена аппроксимация

$$
J(x)=J(x ; \alpha, \beta)=(1-\gamma)\left(\frac{x}{1-x}\right)^{\alpha}, \quad 0 \leqslant x \leqslant \frac{1}{2}, \quad \gamma=\frac{\alpha}{\varepsilon},
$$

для которой, как и для функции бета-распределения, справедлива формула распространения $J(x ; \alpha, \beta)=1-J(1-x ; \beta, \alpha)$, если $\frac{1}{2} \leqslant x \leqslant 1$. Это обстоятельство позволяет нам в дальнейшем ограничиться рассмотрением только области $0 \leqslant x \leqslant \frac{1}{2}$.

В [4] также исследовалась точность аппроксимации $J(x)$. Было показано, что при $\alpha, \beta \leqslant 1$ разность $\delta(x)=I(x)-J(x)$ имеет два экстремума: положительный максимум в точке $x_{1}=1-A^{-1 / \varepsilon}<0.5$ и отрицательный минимум в точке $x_{2}=A^{-1 / \varepsilon}>0.5$, где

$$
A=\frac{\Gamma(1+\varepsilon)}{\Gamma(1+\alpha) \Gamma(1+\beta)}
$$

причем $x_{1}$ доставляет абсолютный максимум функций $|\delta(x)|$ (ошибки $\Delta$ аппроксимации в равномерной метрике), если $\alpha \leqslant \beta$. Интегральное представление

$$
\delta(x)=A \beta \int_{0}^{x} t^{\alpha}(1-t)^{\beta-1} d t
$$

позволило получить ряд возрастающих по величине оценок сверху для ошибки $\Delta$, из которых самая простая и самая грубая такова: $\Delta \leqslant \varepsilon^{2}$. Все эти оценки приведены во введении к таблицам [3], а также в монографии [1] (см. с. 28-29), представляющей обширнейший материал по различным приложениям бета-распределения.

Следует заметить, что формула (1) позволяет также найти асимптотику $(\varepsilon \rightarrow 0)$ ошибки $\Delta$, что не было сделано в [4]. Предполагая в дальнейшем, что $\alpha \leqslant \beta$, и делая достаточно простые вычисления, связанные с разложением в ряд Тейлора, получаем

откуда

$$
x_{1}=\frac{\alpha \beta}{\alpha+\beta} \frac{\pi^{2}}{6}+O\left(\varepsilon^{2}\right)
$$

$$
\Delta=\delta\left(x_{1}\right)=\frac{\alpha \beta^{2}}{\varepsilon} \frac{\pi^{2}}{6}+O\left(\varepsilon^{3}\right) .
$$

Именно такую асимптотику имеют две наиболее точные оценки сверху для значений $\Delta$, указанные в самом начале формулировки основной теоремы статьи [4].

Основная цель нашей статьи - построить ряд уточняющих аппроксимаций $J_{n}(x)$, $n=1,2, \ldots$, бета-распределения, основанных на стохастическом представлении, полученном в начале п. 2 статьи [4], и найти асимптотическое представление для ошибки 
полученной аппроксимации в равномерной метрике. Такая асимптотика позволяет за счет выбора большого значения $n$ сделать главный член асимптотики (2) сколь угодно малым.

2. Построение уточняющих аппроксимаций. В п. 2 статьи [4] было показано, что если $\xi$ - случайная величина с функцией распределения $I(x)$, то преобразование $\xi$ вида $\eta=\varepsilon \ln [\xi /(1-\xi)]$ имеет следующее стохастическое представление:

$$
\eta=\sum_{k=1}^{n}\left(\xi_{2, k}-\xi_{1, k}\right)+\xi_{3, n},
$$

где случайные величины $\xi_{i, k}, k=1, \ldots, n, i=1,2$, и $\xi_{3, n}$ независимы в совокупности, $\xi_{1, k}$ имеет показательное распределение $1-\exp \{-\theta x\}$ с параметром $\theta=(k+\alpha-1) / \varepsilon$, случайная величина $\xi_{2, k}$ также распределена по показательному закону с параметром $\theta=(k+\beta-1) / \varepsilon, k=1, \ldots, n$, а $\xi_{3, n}=\varepsilon \ln \left[\varphi_{n} /\left(1-\varphi_{n}\right)\right]$, где $\varphi_{n}$ имеет бетараспределение с параметрами $\alpha+n$ и $\beta+n$. Таким образом, $\varphi_{n}$ асимптотически $(n \rightarrow \infty)$ нормальна с параметрами

$$
\mathbf{E} \varphi_{n}=\frac{\alpha+n}{\varepsilon+2 n}, \quad \mathbf{D} \varphi_{n}=\frac{(\alpha+n)(\beta+n)}{(1+\varepsilon+2 n)(\varepsilon+2 n)^{2}},
$$

так что при $n \rightarrow \infty$ имеем $\varphi_{n} \rightarrow \frac{1}{2}$, а $\xi_{3, n} \rightarrow 0$ по вероятности.

Наша ближайшая цель - сначала найти распределение случайной величины

$$
\eta_{n}=\sum_{k=1}^{n}\left(\xi_{2, k}-\xi_{1, k}\right),
$$

а затем распределение $J_{n}$ случайной величины $\xi_{n}$, определяемой соотношением $\eta_{n}=$ $\varepsilon \ln \left[\xi_{n} /\left(1-\xi_{n}\right)\right]$. Таким образом мы получим уточнение $J_{n}(x)$ аппроксимации $J_{1}(x)=$ $J(x)$, найденной в статье [4].

Лемма 1. Пусть $\xi_{1}, \ldots, \xi_{n}$ независимы и каждое $\xi_{k}$ имеет показательное распределение с параметром $\theta=(\alpha+k-1) / \varepsilon, k=1, \ldots, n$. Тогда функция распределения их суммь

$$
\begin{aligned}
G_{n}(x, \alpha) & =\mathbf{P}\left(\sum_{k=1}^{n} \xi_{k}<x\right) \\
& =1-\exp \left\{-\frac{\alpha x}{\varepsilon}\right\} \sum_{k=0}^{n-1} \frac{\Gamma(\alpha+k)}{k ! \Gamma(\alpha)}\left(1-\exp \left\{-\frac{x}{\varepsilon}\right\}\right)^{k}
\end{aligned}
$$

Д о к а з а т е л ь с т в о легко провести, применяя метод полной математической индукции и используя очевидное рекуррентное соотношение

$$
G_{n+1}(x, \alpha)=\frac{\alpha}{\varepsilon} \int_{0}^{x} \exp \left\{-\frac{\alpha x}{\varepsilon}\right\} G_{n}(x-t, \alpha+1) d t .
$$

Случай $n=1$ не требует каких-либо вычислений, и предположение, что (3) справедливо для некоторого $n$ приводит к необходимости проверить справедливость соотношения (4) для распределения (3), т.е. доказать равенство

$$
\begin{aligned}
1- & \exp \left\{-\frac{\alpha x}{\varepsilon}\right\}-\frac{\alpha}{\varepsilon} \exp \left\{-\frac{(\alpha+1) x}{\varepsilon}\right\} \sum_{k=0}^{n-1} \frac{\Gamma(k+\alpha+1)}{k ! \Gamma(\alpha+1)} \\
& \times \int_{0}^{x} \exp \left\{\frac{t}{\varepsilon}\right\}\left(1-\exp \left\{-\frac{x-t}{\varepsilon}\right\}\right)^{k} d t \\
= & 1-\exp \left\{-\frac{\alpha x}{\varepsilon}\right\} \sum_{k=0}^{n} \frac{\Gamma(k+\alpha)}{k ! \Gamma(\alpha)}\left(1-\exp \left\{-\frac{x}{\varepsilon}\right\}\right)^{k} .
\end{aligned}
$$


Замена $x-t=s$ упрощает вычисление интеграла в левой части этого равенства, после чего его справедливость устанавливается сдвигом индекса суммирования.

Чтобы упростить интегрирование и вывод асимптотического разложения, удобнее представить функцию $G_{n}$ в виде многочлена от переменной $\exp \{-x / \varepsilon\}$. Для этого в формуле (3) разложим в сумму бином $(1-\exp \{-x / \varepsilon\})^{k}$, изменим порядок суммирования, суммируя сначала по индексу $i$, а потом по $k$, и введем обозначения

$$
a_{n, i}(\alpha)=\sum_{k=i}^{n-1}(-1)^{i} C_{k}^{i} \frac{\Gamma(\alpha+k)}{k ! \Gamma(\alpha)} .
$$

В результате функция распределения принимает вид

$$
G_{n}(x, \alpha)=\sum_{i=0}^{n-1} a_{n, i}(\alpha) \exp \left\{-\frac{(\alpha+i) x}{\varepsilon}\right\} .
$$

Найдем теперь функцию распределения $F_{n}(x)=F_{n}(x ; \alpha, \beta)$ случайной величины $\eta_{n}=\xi_{2}-\xi_{1}$, где $\xi_{1}$ и $\xi_{2}$ независимы и имеют функции распределения $G_{n}(x, \alpha)$ и $G_{n}(x, \beta)$ соответственно (см. (3)). Так как, очевидно,

$$
F_{n}(x ; \alpha, \beta)=1-F_{n}(-x ; \beta, \alpha),
$$

то достаточно рассмотреть только один из случаев $x>0$ или $x<0$.

Лемма 2. Если $x<0$, mo

$$
F_{n}(x)=\sum_{k=0}^{n-1} b_{n, k}(\alpha, \beta) \exp \left\{\frac{(\alpha+k) x}{\varepsilon}\right\},
$$

гдe

$$
b_{n, k}(\alpha, \beta)=a_{n, k}(\alpha) \sum_{i=0}^{n-1} \frac{\beta+i}{\varepsilon+i+k} a_{n, i}(\beta) .
$$

Д о к а 3 а т е л ь с т в о. Пусть $g_{n}-$ функция плотности распределения $G_{n}$. Если $x>0$, то искомое распределение имеет вид

$$
F_{n}(x)=\int_{0}^{\infty} g_{n}(t, \alpha) G_{n}(t+x, \beta) d t=1-\sum_{i=0}^{n-1} \sum_{k=0}^{n-1} \frac{\alpha+i}{\varepsilon+i+k} \exp \left\{-\frac{(\beta+k) x}{\varepsilon}\right\},
$$

поскольку $\sum_{i=0}^{n-1} a_{n, i}(\alpha)=1$.

Мы будем вычислять аппроксимацию $J_{n}(x)$ при $x<\frac{1}{2}$, а этим значениям соответствует область $x<0$ функции $F_{n}(x)$. Применяя соотношение $(5)$ к правой части формулы (8), получаем утверждение леммы.

Последовательность $J_{n}, \quad n=1,2, \ldots$, уточняющих аппроксимаций бетараспределения при $\varepsilon \rightarrow 0$ вычисляется в следующей теореме.

Теорема 1. Если $x<\frac{1}{2}$, mo

$$
J_{n}(x ; \alpha, \beta)=\sum_{k=0}^{n-1} b_{n, k}(\alpha, \beta)\left(\frac{x}{1-x}\right)^{\alpha+k},
$$

где $b_{n, k}(\alpha, \beta)$ вычисляется по формуле $(7)$, и $J_{n}(x ; \alpha, \beta)=1-J_{n}(1-x ; \beta, \alpha)$, когда $x \geqslant \frac{1}{2}$.

Д о к а з а т е л ь с т в о получается простой заменой в формуле (6) переменной $x$ на $\varepsilon \ln [x /(1-x)]$.

Коэффициентам $b_{n, k}=b_{n, k}(\alpha, \beta), k=0,1, \ldots, n-1$, в записи аппроксимации $J_{n}$ можно придать более простой вид, а именно,

$$
b_{n, k}=\frac{(-1)^{k}}{k !(n-1-k) !(\alpha+k)} \prod_{i=0}^{n-1} \frac{(\alpha+i)(\beta+i)}{\varepsilon+k+i} .
$$


Действительно, делая последовательно почленные сложения и применяя метод индукции, легко убедиться, что

$$
a_{n, 0}(\alpha)=\sum_{k=0}^{n-1} \frac{\Gamma(\alpha+k)}{\Gamma(\alpha) k !}=\frac{(\alpha+1)(\alpha+2) \cdots(\alpha+n-1)}{(n-1) !} .
$$

Для произвольного $i=1, \ldots, n-1$ осуществляем следующие преобразования:

$$
a_{n, i}(\alpha)=\frac{(-1)^{i}}{i !} \sum_{k=i}^{n-1} \frac{\Gamma(\alpha+k)}{\Gamma(\alpha)(k-i) !}=\frac{(-1)^{i}}{i !} \sum_{k=0}^{n-1-i} \frac{\Gamma(\alpha+k+i)}{\Gamma(\alpha) k !} .
$$

Теперь к последней сумме применяем те же почленные сложения, что и при выводе формулы (10). Следовательно,

$$
a_{n, i}(\alpha)=(-1)^{i} \frac{\alpha(\alpha+1) \cdots(\alpha+n-1)}{i !(n-1-i) !(\alpha+i)}, \quad i=0,1, \ldots, n-1 .
$$

После подстановки полученных выражений в правую часть (7) доказательство формулы (9) сводится к вычислению следующей суммы:

$$
\begin{aligned}
\sum_{i=0}^{n-1}(-1)^{i} C_{n-1}^{i} \frac{1}{\varepsilon+k+i} & =\sum_{i=0}^{n-1}(-1)^{i} C_{n-1}^{i} \int_{0}^{1} x^{\varepsilon+k+i-1} d x \\
& =\int_{0}^{1} x^{\varepsilon+k-1}(1-x)^{n-1} d x=\frac{\Gamma(\varepsilon+k) \Gamma(n)}{\Gamma(\varepsilon+k+n)} .
\end{aligned}
$$

Приведем несколько первых аппроксимаций $J_{n}(x ; \alpha, \beta)$ бета-распределения в области $x<\frac{1}{2}$ :

$$
\begin{aligned}
J_{1}(x)= & \frac{\beta}{\alpha+\beta}\left(\frac{x}{1-x}\right)^{\alpha}, \\
J_{2}(x)= & \frac{\beta(\beta+1)}{\alpha+\beta+1}\left(\frac{x}{1-x}\right)^{\alpha}\left(\frac{\alpha+1}{\alpha+\beta}-\frac{\alpha}{\alpha+\beta+2} \frac{x}{1-x}\right), \\
J_{3}(x)= & \frac{\beta(\beta+1)(\beta+2)}{\alpha+\beta+2}\left(\frac{x}{1-x}\right)^{\alpha}\left[\frac{(\alpha+1)(\alpha+2)}{2(\alpha+\beta)(\alpha+\beta+1)}\right. \\
& -\frac{\alpha(\alpha+2)}{(\alpha+\beta+1)(\alpha+\beta+3)} \frac{x}{1-x} \\
& \left.+\frac{\alpha(\alpha+1)}{2(\alpha+\beta+3)(\alpha+\beta+4)}\left(\frac{x}{1-x}\right)^{2}\right] .
\end{aligned}
$$

3. Асимптотическое исследование точности аппроксимаций. Точность $\Delta$ аппроксимации $J_{n}$ в равномерной метрике равна наибольшему по абсолютной величине экстремуму функции

$$
\delta(x)=I(x ; \alpha, \beta)-J_{n}(x ; \alpha, \beta), \quad 0 \leqslant x \leqslant 1 .
$$

Исследуем сначала асимптотику точек достижения экстремумов функции $\delta(x)$ на отрезке $\left[0 ; \frac{1}{2}\right]$. Как будет видно в дальнейшем, полученная асимптотика будет соответствовать точке достижения абсолютного макисмума $\delta(\cdot)$, если $\alpha \leqslant \beta$.

Лемма 3. Существует такое $\varepsilon_{0}$, ито при любом $\varepsilon<\varepsilon_{0}$ бункиия $\delta(x)$ на отрезке $\left[0 ; \frac{1}{2}\right]$ имеет единственный максимум в точке

$$
x_{m}=\frac{\alpha \beta}{\varepsilon} n \psi^{\prime}(n)+O\left(\varepsilon^{2}\right)
$$

где $\psi^{\prime}$ - тригамма-функиия Эйлера, при иелочисленном аргументе определяемая формулой

$$
\psi^{\prime}(n)=\frac{\pi^{2}}{6}-\sum_{k=1}^{n-1} \frac{1}{k^{2}}
$$


Д о к а з а т е л ь с т в о. Используя формулу (9) в записи аппроксимации $J_{n}$, представим уравнение $I^{\prime}(x)=J_{n}^{\prime}(x)$, определяющее экстремумы функции $\delta(x)$, в виде

$$
(1-x)^{\varepsilon}=A\left[1+\sum_{k=1}^{n-1}(-1)^{k} C_{n-1}^{k}\left(\frac{x}{1-x}\right)^{k} \cdot \prod_{i=0}^{n-1} \frac{\varepsilon+i}{\varepsilon+k+i}\right],
$$

где

$$
A=\frac{\Gamma(\alpha+n) \Gamma(\beta+n)}{\Gamma(\varepsilon+n) \Gamma(n)}=\exp \left\{-\alpha \beta \psi(n)+O\left(\varepsilon^{3}\right)\right\} .
$$

Данная асимптотика величины $A$ и вид уравнения (12) показывают, что при $\varepsilon \rightarrow$ 0 точка достижения максимума $x_{m}$ стремится к 0 . Следовательно, уравнение $(12)$ можно представить в асимптотическом виде

$$
(1-x)^{\varepsilon}=A\left(1-\varepsilon \frac{n-1}{n} x+O\left(x^{2}\right)\right) .
$$

Логарифмируя обе части этого уравнения и применяя асимптотическую формулу $\ln (1+x)=x+O\left(x^{2}\right)$, находим, что $\ln A+\varepsilon x / n+O\left(x^{2}\right)=0$, откуда непосредственно следует утверждение леммы.

Используя формулу распространения, нетрудно убедиться, что при $\varepsilon \rightarrow 0$ функция $\delta(x)$ в области $\frac{1}{2}<x \leqslant 1$ обладает отрицательным минимумом в точке $x=1-x_{m}$.

Точность аппроксимации $J_{n}$ устанавливает следующая теорема.

Теорема 2. Если $\varepsilon \rightarrow 0$, mo

$$
\Delta=\max _{0<x<1}|\delta(x)|=\frac{\alpha \beta}{\varepsilon} \psi^{\prime}(n) \cdot \max \{\alpha, \beta\}+O\left(\varepsilon^{3}\right) .
$$

Д о к а з а т е л ь с т в о. Если $0<x<\frac{1}{2}$, то при $\alpha \leqslant \beta$ максимум ошибки аппроксимации достигается в точке $x_{m}<\frac{1}{2}$. Следовательно,

$$
\begin{aligned}
\Delta_{(0,1 / 2)}= & \frac{1}{\Gamma(n)} \prod_{i=0}^{n-1} \frac{(\alpha+i)(\beta+i)}{\varepsilon+i} \\
& \times \int_{0}^{x_{m}}\left[\frac{(1-x)^{\varepsilon}}{A}-1-\sum_{k=1}^{n-1}(-1)^{k} C_{n-1}^{k}\left(\frac{x}{1-x}\right)^{k} \cdot \prod_{i=0}^{n-1} \frac{\varepsilon+i}{\varepsilon+k+i}\right] \\
& \times\left(\frac{x}{1-x}\right)^{\alpha-1} d\left(\frac{x}{1-x}\right) .
\end{aligned}
$$

Выражение под интегралом, стоящее в квадратных скобках, обращается в нуль, когда $x=x_{m}$, и поэтому интегрирование по частям (сомножитель под интегралом, стоящий после квадратных скобок, берется за $d v)$ дает

$$
\begin{aligned}
\Delta_{(0,1 / 2)}= & \frac{1}{\Gamma(n)} \prod_{i=0}^{n-1} \frac{(\alpha+i)(\beta+i)}{\varepsilon+i} \cdot \frac{1}{\alpha} \int_{0}^{x_{m}}\left(\frac{x}{1-x}\right)^{\alpha}\left[\frac{\varepsilon(1-x)^{\varepsilon-1}}{A}\right. \\
& \left.+\frac{1}{(1-x)^{2}} \sum_{k=1}^{n-1}(-1)^{k} C_{n-1}^{k} k\left(\frac{x}{1-x}\right)^{k-1} \cdot \prod_{i=0}^{n-1} \frac{\varepsilon+i}{\varepsilon+k+i}\right] d x .
\end{aligned}
$$

Разложение Тейлора по степеням $x_{m}$ с сохранением главных асимптотических членов показывает, что

$$
\Delta_{(0,1 / 2)}=\frac{\alpha \beta^{2}}{\varepsilon} \psi^{\prime}(n)+O\left(\varepsilon^{3}\right) .
$$

Аналогичные вычисления в области $\frac{1}{2}<x<1$ дают

$$
\Delta_{(1 / 2,1)}=\frac{\alpha^{2} \beta}{\varepsilon} \psi^{\prime}(n)+O\left(\varepsilon^{3}\right) .
$$


Теорема доказана.

Поскольку при $n \rightarrow \infty$ справедлива асимптотика $\psi^{\prime}(n) \sim n^{-1}$, то использование $J_{n}$ вместо $J_{1}$ увеличивает точность аппроксимации приблизительно в $n$ раз.

В таблице 1 приводятся точные значения величины $\Delta$ и ее асимптотики (в скобках), указанные в формулировке теоремы 2. Из данных таблицы можно заметить, что на точность аппроксимации влияют не столько большие значения $n$, сколько малые значения параметров $\alpha$ и $\beta$.

Таблица 1. Ошибки аппроксимации $J_{n}$

\begin{tabular}{|c|c|c|c|}
\hline$\imath$ & $\alpha=0.5, \beta=0.05$ & $\alpha=0.1, \beta=0.5$ & $\alpha=0.01, \beta=0.5$ \\
\hline 1 & $(0.205617)$ & $(0.068539)$ & $(0.008063)$ \\
\hline 2 & $(0.080617)$ & $(0.026872)$ & $(0.003161)$ \\
\hline 3 & $(0.049367)$ & $(0.016456)$ & $(0.001936)$ \\
\hline 5 & $(0.027665)$ & $(0.009222)$ & $(0.001085)$ \\
\hline 7 & 0.005876 & 0.004434 & $(0.000753)$ \\
\hline & $(0.013146)$ & $(0.004382)$ & 0.000476 \\
\hline & $\alpha=0.001$ & $\alpha=10^{-4}, \beta$ & $\alpha=0.1, \beta$ \\
\hline ] & $(0.000821)$ & $(0.000082)$ & $(0.008225)$ \\
\hline V & 0.000277 & $(0.000032)$ & 0.002096 \\
\hline 3 & 0.000179 & $(0.000020)$ & $(0.001975)$ \\
\hline 5 & 0.000104 & 0.000010 & 0.000737 \\
\hline 7 & $(0.000077)$ & $(0.000008)$ & 0.000513 \\
\hline & $(0.000052)$ & $(0.000005)$ & $(0.000526)$ \\
\hline & $\alpha=0.01$ & $0.001, \beta$ & $10^{-4}$ \\
\hline & $(0.001495)$ & $(0.000163)$ & $(0.000008)$ \\
\hline 2 & $(0.000589)$ & $(0.000064)$ & 0.000006 \\
\hline 3 & $(0.000359)$ & 0.000038 & $(0.000004)$ \\
\hline 5 & 0.000188 & 0.000022 & $(0.000002)$ \\
\hline & $(0.000140)$ & 0.000015 & $(0.000002)$ \\
\hline & $(0.000096)$ & 0.000010 & 0.000001 \\
\hline & $\alpha=0.01$ & $\alpha=0.001, \beta=0.01$ & $10^{-4}$ \\
\hline & $(0.000082)$ & $(0.000015)$ & $(0.000002)$ \\
\hline & $(0.000032)$ & $(0.000006)$ & $(0.000001)$ \\
\hline & $(0.000020)$ & $(0.000004)$ & $(0.000000)$ \\
\hline & $(0.000011)$ & 0.000002 & $(0.000000)$ \\
\hline & $(0.000008)$ & 0.000001 & 0.000000 \\
\hline & $(0.000005)$ & 0.000001 & $(0.000000)$ \\
\hline
\end{tabular}

\section{СПИСОК ЛИТЕРАТУРЫ}

1. Gupta A. K., Nadarajah S. (Eds.). Handbook of Beta Distribution and its Application. New York: Dekker, 2004, 571 p.

2. Pearson K. Tables of the Incomplete Beta Function. London: Cambridge Univ. Press, $1968,505 \mathrm{p}$

3. Пирсон К. Таблицы неполной бета-функции. М.: ВЦ АН СССР, 1974, 540 с.

4. Володин И. Н. Бета-распределение при малых значениях параметров. - Теория вероятн. и ее примен., 1970, т. 15, в. 3, с. 563-566.

Поступила в редакцию 29.III.2006

Исправленный вариант 14.IV.2009 